\title{
An unsupervised machine learning approach to extract wheel and track health status indicators from train-borne accelerometer data
}

\author{
BENJAMIN BAASCH ${ }^{1}$, MICHAEL ROTH ${ }^{2}$, SEBASTIAN SCHULZ ${ }^{3}$ and JÖRN C. \\ GROOS $^{4}$ \\ ${ }^{1}$ Institute of Transportation Systems, German Aerospace Center (DLR), Rutherfordstr. 2, \\ 12489 Berlin, Germany. \\ E-mail: benjamin.baasch@dlr.de \\ ${ }^{2}$ Institute of Transportation Systems, German Aerospace Center (DLR), Lilienthalplatz 7 , \\ 38108 Braunschweig, Germany. \\ E-mail: m.roth@dlr.de \\ ${ }^{3}$ Institute of Transportation Systems, German Aerospace Center (DLR), Rutherfordstr. 2, \\ 12489 Berlin, Germany. \\ E-mail: s.schulz@dlr.de \\ ${ }^{4}$ Institute of Transportation Systems, German Aerospace Center (DLR), Lilienthalplatz 7 , \\ 38108 Braunschweig, Germany. \\ E-mail: joern.groos@dlr.de
}

Within the project SIA (System for vehicle-infrastructure interaction assets health status monitoring) the German Aerospace Center (DLR) is developing and testing a low-cost multisensor system for monitoring the health status of the wheel and rail. This sensor-system is based on components-off-the-shelf (COTS) suitable for the application on in-service trains (Baasch et al., 2018). The system gathers vibrations at the wheelset with a high-bandwidth $(0.8$ to $8000 \mathrm{~Hz})$ three-component Axle-Box Acceleration (ABA) sensor. Wheel and rail roughness are the main influencing factors for vibrations of the wheel set. In this study, a blind signal separation (BSS) algorithm based on non-negative matrix factorization is used to simultaneously extract features from ABA data related to the wheel and the rail health status. The algorithm uses unsupervised machine learning and hence does not rely on labelled data, a-priori knowledge or physical models. It makes use of different time-frequency characteristics of the vibration signals and is thus able to separate signal components related to the wheel health status from those related to the rail health status. Through georeferencing the exact localization of the extracted features can be mapped on the track. Additionally, the BSS algorithm reduces the dimensionality of the data without significant loss of information. The presented approach was successfully applied to data measured on a European railway mainline with a modified passenger train at normal operation's speed.

Keywords: machine learning, dimensionality reduction, blind signal separation, condition monitoring, condition-based maintenance, axle-box acceleration.

\section{References}

Baasch, B., Roth, M. and Groos, J. (2018). In-service condition monitoring of rail tracks: On an onboard low-cost multi-sensor system for condition based maintenance of railway tracks. Internationales Verkehrswesen, vol. 70, no. 1, pp. 76-79 